\title{
Tendencias de la innovación tecnológica en Colombia 1991-2013 a partir del análisis de patentes
}

\author{
Rosa Elena Gómez Hurtado*
}

Artículo recibido:

19 de octubre de 2017

Artículo aceptado:

20 de septiembre de 2018

Artículo de investigación

\section{RESUMEN}

Se presenta un análisis de las patentes concedidas a instituciones o inventores de origen colombiano registradas en las bases de $\mathrm{d}$ atos de las oficinas de patentes de Estados Unidos, Europa, WIPO, Alemania y Colombia. Se observan las tendencias de innovación tecnológica que rigen los procesos y productos en Colombia durante el periodo y se comprueba que efectivamente las patentes son indicadores de innovación tecnológica. Se evidencia que las invenciones de colombianos no son necesariamente patrocinadas por instituciones colombianas y que la figura del inventor solitario tiende a desaparecer para dar paso a las innovaciones industriales en manos de los equipos

\footnotetext{
Facultad de Ingeniería y Nuevas Tecnologías

Fundación Universitaria UNINPAHU, Colombia_rhgomez02@uninpahu.edu.co

INVESTIGACIÓN BIBLIOTECOLÓGICA, vol. 32, núm. 77, octubre/diciembre, 2018, México, ISSN: 2448-8321 pp. 133-150
} 
de investigación de las grandes empresas, conviertiéndose en un mecanismo de control de la producción y comercialización mundiales.

Palabras clave: Innovación Tecnológica; Patentes; Patentometría

\title{
Patent analysis of Colombian technological innova-
} tion 1991 - 2013

Rosa Elena Gómez-Hurtado

\begin{abstract}
This study offers an analysis of patents granted to Colombian institutions or inventors and registered in patent databases in the US, Europe, WIPO, Germany and Colombia. The trends regarding processes and products of technological innovation in Colombia during the period are observed showing that patents are in fact indicators of technological innovation. Moreover, Colombian inventions are not necessarily sponsored by Colombian institutions, and the and phenomenon of the solitary inventor is giving way to research teams working for large companies, which exercise control over production and marketing.
\end{abstract}

Keywords: Technological Innovation; Patents; Patentometrics

\section{INTRODUCCIÓN}

$\mathrm{E}^{1}$ lanálisis de patentes es una de las herramientas para entender y direccionar el desarrollo tecnológico y la innovación, pero por sí solas las patentes no desencadenan el proceso de desarrollo tecnológico, pues forman parte de un sistema dinámico en el cual los esfuerzos que se le dediquen a la I+D serán la materia prima del sistema científico tecnológico (Montes Hincapié et al., 2009). Esta es una de las razones por las que se monitorean las actividades de ciencia, tecnología e innovación (ACTI) al igual que por la incidencia que el patentamiento tiene en las estrategias de $\mathrm{I}+\mathrm{D}$ en el desarrollo de los países. 
El periodo analizado (1991-2013) tiene características determinantes que es necesario tener presentes por su incidencia en la decadencia de áreas que para los inventores eran muy importantes, pero que con el paso del tiempo van perdiendo interés y son reemplazadas por otras que se convierten en estratégicas; es allí donde se concentra el nivel de I+D+I. Para el patentamiento, la globalización de la economía, caracterizada por la complejidad y un alto nivel de incertidumbre, obligó a las empresas a ser más competitivas, a centrarse en su saber hacer y a establecer las alianzas estratégicas necesarias para tener presencia en los mercados globales, con el propósito de reducir la incertidumbre.

Otro hito importante a considerar radica en los cambios tecnológicos que desde décadas pasadas se venían preparando para alcanzar el mayor grado de madurez, a partir de las siguientes características:

- el proceso de fabricación flexible presiona sobre la innovación tecnológica,

- se da una visión orgánica de la empresa, y

- comienzan a desarrollarse estrategias tecnológicas.

La innovación tecnológica es entendida como "el producto, proceso o metodología que aparece en un mercado determinado y que es aceptado por el mismo" (Barceló, Solé Parellada y Valls, 1992: 127). Sin embargo, es conveniente aclarar que innovación no es sinónimo de invención, sino un concepto o proceso más amplio (Ortiz y Pedroza Zapata, 2006: 66). En este contexto, las actividades de innovación tecnológica son el conjunto de etapas científicas, tecnológicas, organizativas, financieras y comerciales, incluyendo las inversiones en nuevos conocimientos, que llevan o intentan llevar a la implementación de productos y de procesos nuevos o mejorados (OCDE, 2003: 17). Estas actividades son la adquisición de la tecnología y de know-bow, la puesta a punto de herramientas y la ingeniería industrial, entre otras. $\mathrm{Nu}$ merosos estudios han demostrado que las actividades de $\mathrm{I}+\mathrm{D}$ se realizan en cooperación con investigadores individuales, equipos de investigadores y unidades de investigación.

En la actividad inventiva el indicador más elemental y generalmente más utilizado es el simple cómputo de las patentes, aunque también se pueden contabilizar aquellas registradas por los inventores de un país determinado, bien sea en forma global o por sectores de actividad. Este indicador debe ser manipulado con precaución, puesto que las estrategias de adopción de patentes dependen de los sectores, de las compañías y de las legislaciones nacionales (Callon, Courtial y Penan, 1995). 
En Colombia, el modelo lineal de la ciencia (insumo-producto) que sigue las orientaciones del Manual de Frascati evalúa el gasto que tienen las empresas en ACTI a partir de los resultados de las encuestas de desarrollo e innovación tecnológica (EDIT), distinguiendo aquellas actividades dedicadas a la innovación (Lucio et al., 2013), teniendo en cuenta que están atadas al fortalecimiento del Sistema Nacional de Ciencia, Tecnología e Innovación (SNCTI) (CONPES, 2008) a través de acciones como la mejora de la innovación tecnológica con énfasis en los sectores de clase mundial y la promoción de la productividad y la competitividad teniendo como plataforma la ciencia, la tecnología y la innovación.

En este sentido, el análisis de la política de innovación colombiana, que debe ir más allá del desarrollo científico y tecnológico, muestra que las empresas colombianas están siendo cada vez menos innovadoras y el gasto en actividades de I+D es demasiado bajo (70 \% del PIB) (BID, 2014: 9).

Es así que la innovación tecnológica se constituye en la base de la competitividad y en una mayor integración de los productos, los procesos y el mercado, lo que determina la dinámica de patentación de los inventos de los colombianos en el periodo de estudio, principalmente a mediados de ese lapso, cuando la dinámica de patentación de innovación tecnológica cambia.

El artículo se estructura en tres partes: en la primera, se introduce el tema a través de un marco general que permite entender y ubicar el objeto de estudio; en la segunda, se presenta la metodología utilizada para el desarrollo de la investigación; en tercer lugar, se aborda el análisis de los resultados, la discusión y las conclusiones.

\section{Metodología}

La investigación, de corte descriptivo, utilizó métodos patentométricos para explorar la innovación tecnológica en Colombia durante el periodo 1991-2013, con el propósito de mostrar el impacto de las patentes como indicadoras de la innovación tecnológica. Estos métodos han sido utilizados para mostrar el rendimiento de la investigación de un país y su competitividad (Huang, Chang y Chen, 2012). Su objetivo consistió en identificar las tendencias de innovación tecnológica en Colombia mediante el análisis de la información contenida en las patentes concedidas a empresas o innovadores colombianos, registrada en los bancos de patentes, durante el periodo señalado.

Se analizaron 5001 patentes concedidas a empresas o a inventores colombianos, independientemente de si la patente fue concedida en el país o en el exterior. La estrategia consistió en recuperar las patentes donde al menos uno 
de los inventores fuera de origen colombiano, utilizando el software AclaimpIP y la base de datos Thomson Innovation que incluye los bancos de patentes de Estados Unidos, Europa, WIPO, Alemania y Colombia, recurriendo a diferentes filtros; al igual que el software Vantage Point. Se trabajó con la clasificación internacional de patentes (IPC) la cual se divide en 8 secciones, cada una de las cuales es designada por una letra mayúscula y se constituye en el nivel jerárquico más alto de la misma; 120 clases y 628 subclases, con el fin de facilitar al investigador su búsqueda y la recuperación de los contenidos de las patentes.

En la primera etapa de la metodología se seleccionaron las fuentes de los datos originales y la descarga de la información se realizó a una hoja de Excel, observando los títulos recuperados y los resúmenes disponibles de cada patente para su posterior tratamiento importando los datos al software Vantage Point, que permite realizar Tech Mining, una forma de analizar contenidos mediante la cual se extrae información útil de varias formas de datos, con énfasis en el análisis de los datos numéricos y explotando especialmente las fuentes de datos de texto estructurado en campos como autor, fecha de publicación y palabras clave, con moderada facilidad en fuentes de texto semiestructuradas (etiquetadas) y utilidad limitada con textos no estructurados (Zhou et al., 2014).

Para localizar los trabajos publicados en el periodo de análisis entre cuyos innovadores, empresas o personas al menos uno fuera colombiano, se efectuó una estrategia de búsqueda global debido a la gran variedad de nombres bajo los cuales aparecen las instituciones en la base de datos. Utilizando la búsqueda avanzada, se estructuró la estrategia de búsqueda de la siguiente manera:

$$
\mathrm{CU}=(\text { Colombia OR Columbia }) \text { AND PY=1991-2013 }
$$

donde CU corresponde al país (se incluye la palabra Columbia porque otros estudios anteriores han detectado instituciones colombianas con el país escrito en esa forma. En este sentido, si bien es cierto que se ha ido corrigiendo progresivamente este error, aún se muestra al país escrito como Columbia en muchos registros) y PY se refiere al año de publicación.

- Una vez subida la información a Vantage Point, se procedió a crear un tesauro que facilitara el tratamiento y filtrado de los datos para superar las posibles discrepancias de los nombres, utilizando rutinas de coincidencia difusa a través de reglas de correlación, como la coocurrencia de términos que indican una posible relación entre los mismos, para fi- 
nalmente consolidar las variaciones en el nombre de las organizaciones o autores, eliminar los registros duplicados, normalización de campos, eliminación o inclusión de caracteres, desagregación de ciertos campos como las direcciones y las categorías de materia, y la unificación de variantes en nombres de instituciones, ciudades, países, entre otros.

- El análisis de los conglomerados, por otra parte, se pudo realizar mediante la agrupación de términos.

- La aplicación del mapeo se basó en una serie de pasos, entre los que se contempló la homogeneización y depuración de la información (confección de un diccionario de términos, eliminación de registros duplicados), obtención de matrices de coocurrencia (palabras, empresas, innovadores, códigos de clasificación de patentes, palabras claves, etc.) y la obtención de mapas tecnológicos. La representación de la información con la utilización del mapeo tecnológico tuvo como fin identificar áreas de conocimiento y obtener una visión de la estructura de relaciones existentes. La distancia en el mapa entre dos palabras (referentes a tecnologías, productos, autores, empresas) señala la mayor o menor relación entre ellas, según van Raan (citado por Escorsa, Maspons y Llibre, 2001).

- El análisis de los resultados se realizó adaptando la metodología propuesta por Goodman y Lawless (1994), ya aplicada por Castellanos et al. (2005). En este punto, el análisis de las fechas de presentación y aprobación de las patentes permitió identificar las tendencias respecto a las áreas de conocimiento, quiénes trabajan en ella y con qué (Tech Mining) (Zhou et al., 2014)

\section{Patentes}

Para efectos de este trabajo, entendemos como patente "una autorización legal que concede a su titular el derecho exclusivo de controlar el uso de una invención, según se la define en sus reivindicaciones, dentro de un área geográfica y en un plazo limitado impidiendo que terceros, entre otras actividades, fabriquen, usen, vendan u ofrezcan en venta la invención sin la autorización pertinente" (OMPI, 2017: 6).

Los documentos de patentes ofrecen diversa información que puede considerarse de dos tipos: 1) la documentación administrativa, que distingue elementos como a) los datos relativos al solicitante y de su representante, b) la documentación aportada, c) datos sobre la vida administrativa de la patente y d) los pagos realizados, y 2) la información técnica integrada por 
tres partes: a) la descripción: plantea el problema técnico que la invención pretende solucionar, a la vez que se detalla el contenido y las características técnicas de la misma, b) las reivindicaciones: se hace referencia a la segunda parte del documento de la patente, y c) los dibujos, que no tienen por qué incluirse siempre, aunque permiten tener una concepción visual más completa de la invención.

Las patentes se constituyen en una de las fuentes de información tecnológica más completas, accesibles, confiables, actualizadas y que abarcan un gran número de sectores, y es por ello que, sobre la base de las reivindicaciones, los examinadores de las oficinas de patentes las catalogan en una categoría de clasificación. Existen varias clasificaciones de patentes y una de ellas es la clasificación internacional de patentes basada en el tratado multilateral internacional administrado por la OMPI (Arreglo de Estrasburgo, 1971), que entró en vigor en 1987 y que en la práctica es utilizada por las oficinas de propiedad industrial de más de 100 países en virtud del Tratado de Cooperación en Materia de Patentes. Sin embargo, aunque sea de aplicación en todo el mundo, algunas oficinas adoptan clasificaciones propias como es el caso de la UPSTO (Oficina de Patentes y Marcas de Estados Unidos) y de la Oficina Europea de Patentes (EPAT).

En el manual de Frascati se indica que ya las estadísticas de $\mathrm{I}+\mathrm{D}$ no son suficientes para evaluar sus resultados y que en el contexto de la economía del conocimiento los datos deben ser examinados en un marco conceptual que permita relacionarlos con otros medios disponibles y con los resultados derivados de las actividades de I+D de que se trate (OCDE, 2003). Es así que el tema de los indicadores para evaluar la ciencia y tecnología surge con la aparición de la tecnometría, desarrollada por Jacob Schmookler en 1950, quien construyó e interpretó por primera vez las estadísticas de patentes para la medición de las actividades científicas y tecnológicas (Orozco y Chavarro, 2010) que se consolidaron en la década de 1960 con la National Science Fundation (NSF) y la OCDE.

Los indicadores de patentes proveen información sobre los resultados de la actividad científica en una institución, país o región del mundo. Tradicionalmente se han clasificado como sigue: a) indicadores de actividad, que proporcionan datos acerca del volumen e impacto de las actividades de investigación y cuyas variables más importantes son los inputs (recursos puestos en circulación) y los outputs o productos que se elaboran, entre los que se encuentran las patentes, y b) indicadores de relación, que rastrean los lazos y las interacciones entre investigadores y campos.

En la actividad inventiva, la patente es examinada por la oficina en don- 
de se presenta la petición teniendo en cuenta la novedad y el contenido de la descripción propuesta, es decir, la reproductibilidad; con lo cual queda claro que la patente describe la invención, pero no la innovación ya que ésta supone la comercialización con éxito. Autores como Spinak (1998), Callon, Courtial y Penan (1995) y Camps (2008) señalan que los indicadores más utilizados son:

- Indicadores de actividad (número de patentes, distribución, países, etc.),

- Indicadores relacionales de primera generación (colaboraciones o vínculos entre inventores, empresas, etc.),

- Indicadores relacionales de segunda generación (coocurrencia de textos, resumen, título, etcétera),

- Indicadores de tercera generación (clasificación de la información mediante clusters, mapas tecnológicos),

- Familia de patentes (permite medir el nivel de actividad tecnológica),

- Identificación de autores, entidades, países y sectores líderes, así como su impacto,

- Identificación de competidores, posibles socios, etc., evolución de la técnica en el sector, caracterización de las empresas involucradas en el sector teniendo en cuenta su comportamiento,

- Impacto y análisis de las citaciones y cocitaciones, y

- Determinación de las principales tendencias tecnológicas e identificación de señales débiles.

\section{Presentación y análisis de Resultados}

El abandono de una patente lleva a que ésta sea de dominio público y, por lo tanto, resulte de libre disposición para cualquier interesado, situación atractiva para aquellas empresas con menos recursos, las cuales pueden detectar tecnología de dominio público que seguramente les puede ser de utilidad.

Sobre esa base, los resultados muestran que el mayor número de patentes no renovadas se presenta en el año 2007 seguido del 2011 y 2013.

En 2003 se observa una disminución de casi el 50 \% de las patentes renovadas, abandono que va disminuyendo paulatinamente a medida que avanzan los años. A diferencia de la tendencia observada en la Figura 1 la supervivencia de patentes presenta una curva ascendente principalmente del $2004 \mathrm{al}$ 2012, y en adelante su comportamiento es estable. 

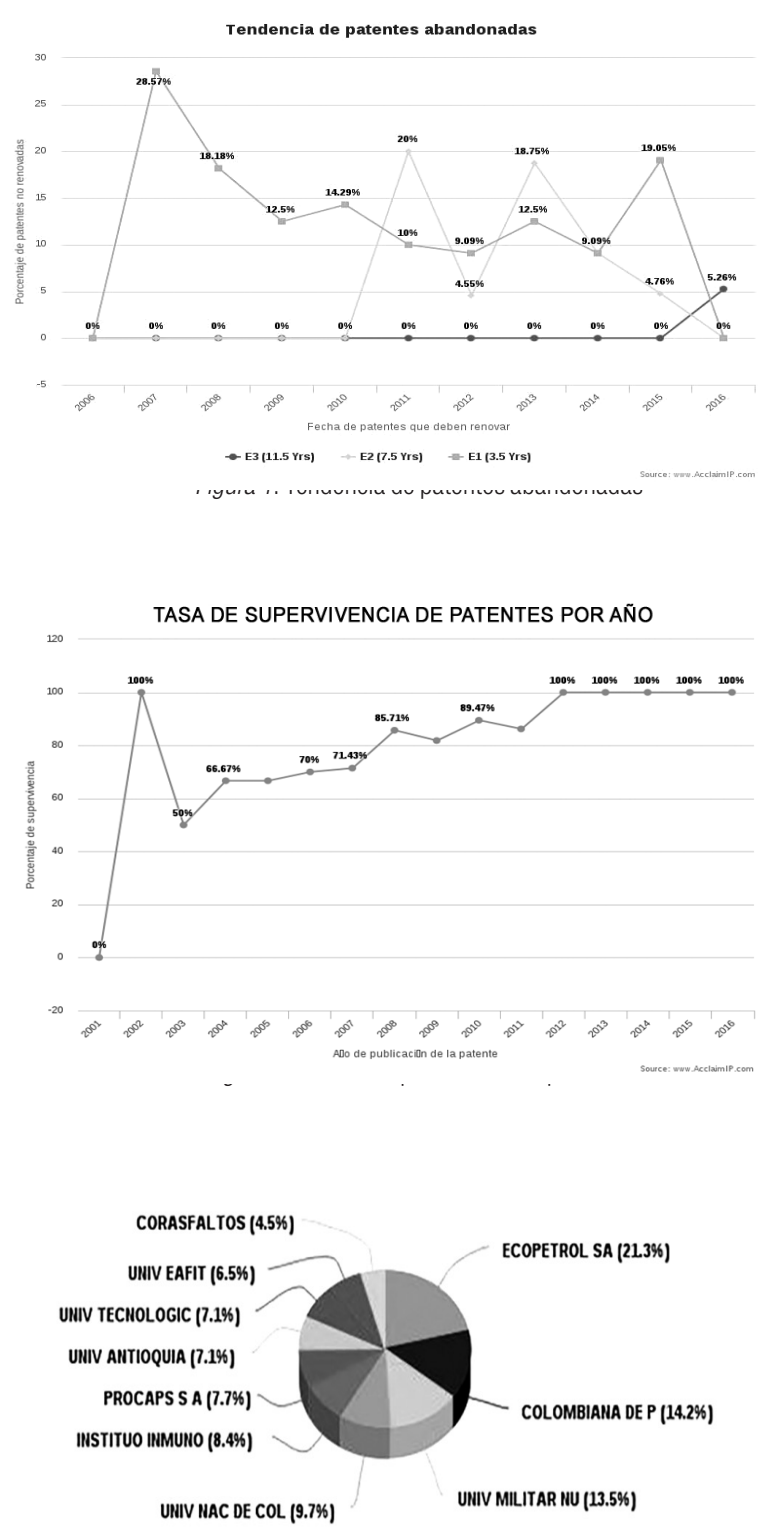
Ecopetrol SA, Colombiana de Petróleos, Universidad Militar Nueva Granada, Universidad Nacional de Colombia, Instituto Inmunología de la Universidad del Valle, PROPCAPS S.A., Universidad de Antioquia y Universidad Tecnológica de Pereira, Universidad EAFIT y CORASFALTOS. Es probable que las instituciones con mayor número de patentes también tengan líneas de investigación muy específicas.

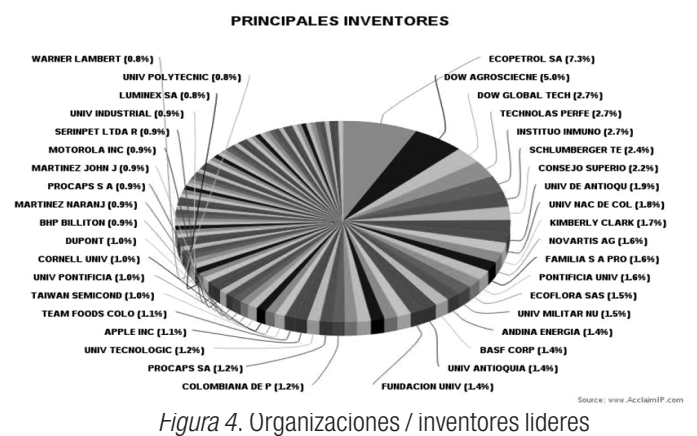

En la Figura 4 se aprecia que ECOPETROL ostenta el liderazgo de la producción total de patentes del país con 145 patentes. Tiene tres patentes conjuntas con la Universidad de Antioquia, tres con EAFIT y cinco con la Universidad Pontificia Bolivariana. Le siguen las empresas Dow Agrosciences LLC con 99 patentes y Dow Global Technology LLC con 49. Con un porcentaje menos significativo se encuentran la empresa Schlumberger Technology, la Universidad de Antioquia, Kimberly Clark y la Pontificia Universidad Javeriana. El $91.1 \%$ restante está compuesto por instituciones o personas naturales, que si bien es cierto tienen menor número de patentes, no por ello son menos importantes.

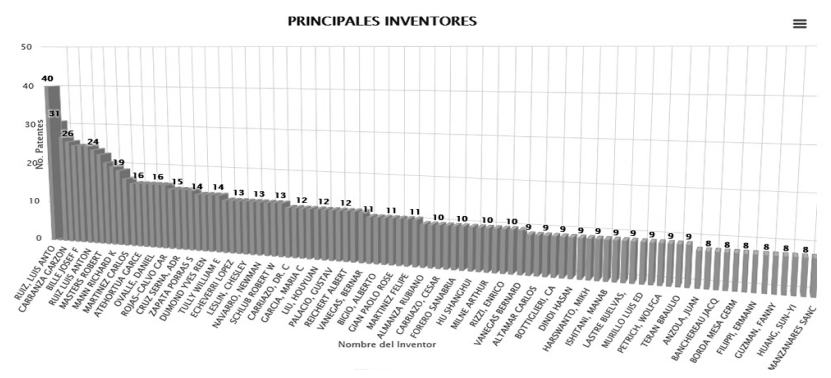

Figura 5. Principales inventores 
La dinámica de la productividad de los inventores permite visualizar que Luis Antonio Ruíz, Josef Bille, Jose Orlando Giraldo, Nelson Carranza Garzón, Robert Master y Richard Mann son quienes mayor cantidad de patentes registran. El promedio de publicación de patentes por las principales clases, según la clasificación internacional de patentes (Tabla 1), muestra una apuesta explícita hacia el sector de las tecnologías para el manejo de residuos sólidos, preparaciones medicinales que contienen ingredientes orgánicos activos y generación de energía a través de fuentes de energía renovables, que son de las que mayor preponderancia tienen para el país en sus dos vertientes: la generación de energía y las fuentes de generación de la misma. En menor medida se encuentran las patentes sobre tratamiento de agua residual o de alcantarilla, alimentos o productos alimenticios, procesamiento de hidrocarburos, tecnologías para la producción de combustible de origen no fósil, y café: substitutos y preparaciones, estas últimas patentes basadas en un producto en el que tradicionalmente el país ha sido líder como productor.

\begin{tabular}{|l|l|}
\hline \multicolumn{1}{|c|}{ CLASE } & \multicolumn{1}{c|}{ DESCRIPCIÓN } \\
\hline Y02W30 & Tecnologías para el manejo de residuos sólidos \\
\hline A23L1 & Alimentos o productos alimenticios. Preparación o tratamiento \\
\hline A61K31 & Preparaciones medicinales que contienen ingredientes activos orgánicos \\
\hline Y02E10 & La generación de energía a través de fuentes de energía renovables \\
\hline A61K9 & Preparaciones medicinales caracterizadas por la forma física especial \\
\hline C02F1 & El tratamiento de agua, agua residual o de alcantarilla \\
\hline Y02E50 & Tecnologías para la producción de combustible de origen no fósil \\
\hline A61K45 & $\begin{array}{l}\text { Preparaciones medicinales que contienen ingredientes activos no previs- } \\
\text { tos en los grupos A61K31/00 to A61K41/00 }\end{array}$ \\
\hline C10G2300 & $\begin{array}{l}\text { Aspectos relacionados con el procesamiento de hidrocarburos cubiertos } \\
\text { por los grupos C10G1/00 - C10G99/00 }\end{array}$ \\
\hline
\end{tabular}

Tabla 1. Principales clases de patentes (grupo principal)

Es importante aclarar que a pesar de que la fecha de presentación y la fecha de prioridad se usan indistintamente, no son lo mismo. La fecha de presentación es cuando una solicitud de patente se presenta por primera vez en una oficina de patentes. La fecha de prioridad establece la novedad y/o la evidencia de una invención en particular en relación con otras y puede ser anterior a la fecha de presentación efectiva de la solicitud. 


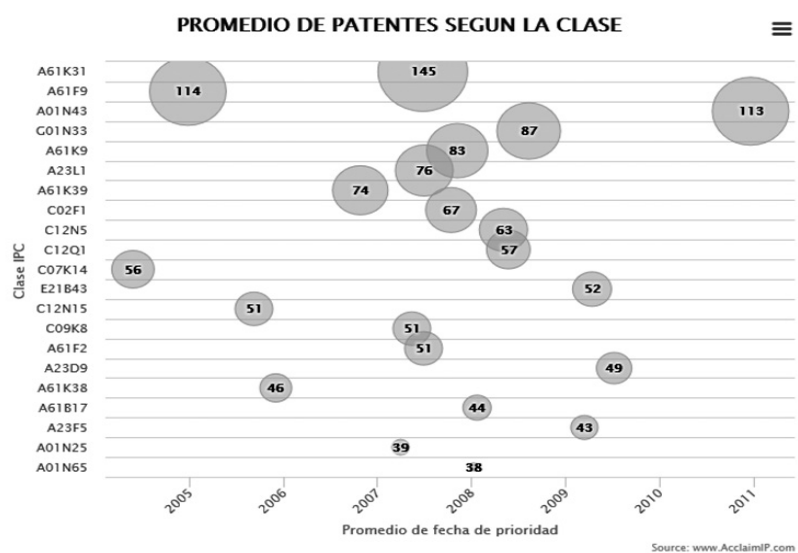

Figura 6. Tendencias tecnológicas según la clase

Ahora bien, si se observa la Figura 6, la tendencia dominante en la producción de patentes se presenta en el 2007 en el campo de preparaciones medicinales que contienen activos orgánicos, mientras que en el año 2004 se orienta a métodos o dispositivos para tratamiento ocular: lentes de contacto, dispositivos para corregir la visión, aparatos para guiar a los ciegos y dispositivos para proteger los ojos. Finalmente, en el 2010 las invenciones giran en torno a las biocidas, repelentes de plagas y reguladores del crecimiento de las plantas que contienen compuestos heterocíclicos.

En este periodo y los subsiguientes se pone en evidencia el intento de los inventores por ser más competitivos, por esta razón, la cooperación universidad-empresa se presenta con muchas posibilidades. Igualmente, el patentamiento y posicionamiento de los productos de patentes en el mercado tienen un costo relacionado con la adquisición de licencias, tecnologías o vigilancia tecnológica, entre otras, y es posible que estos asuntos no estén al alcance de los inventores, por lo cual la alternativa es la realización de alianzas estratégicas con otras empresas para efectuar desarrollos conjuntos como parte de la estrategia empresarial.

En consecuencia, al analizar las subclases se encuentra que a medida que avanzan los años la producción de patentes se concentra en nuevas tecnologías o áreas que bien podrían denominarse emergentes y que antes del 2009 no eran objeto de patentación por parte de los inventores. Surge a partir de ese año el interés de los inventores por inventos relacionados con productos y procedimientos químicos, como preparaciones para propósitos de tocador, aprovechando quizá el esfuerzo propio de I+D y la capacidad investigadora de centros externos constituidos por empresas o instituciones, entre las que 
se encuentran las universidades. A la par, son importantes las patentes relacionadas con alimentos, productos alimenticios o bebidas no alcohólicas y biocidas, repelentes de plagas y reguladores del crecimiento de las plantas que contienen compuestos heterocíclicos.

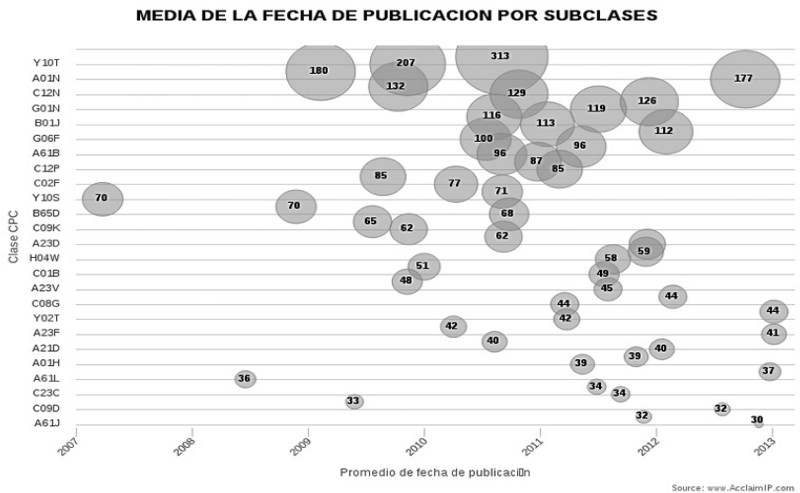

Figura 7. Tendencias y áreas emergentes por subclases

\section{Red de colaboración}

La dinámica de generación de la innovación tecnológica se puede evaluar a partir de las redes de colaboración entre instituciones e innovadores, quienes están unidos por vínculos laborales y académicos. El componente gigante de la red de colaboración durante el periodo 1991-2013 está formado por 226 solicitantes, todos interconectados de algún modo.

El análisis se efectúa a partir de los conceptos de centralidad y densidad, que resaltan la contribución de los diferentes agregados a la estructuración de la red general. Desde esta perspectiva, se observa que entre más numerosas y fuertes sean las relaciones con otros agregados, más se detecta un conjunto de temas de innovación tecnológica considerado crucial para la comunidad de científicos o tecnólogos (Callon, Courtial y Penan, 1995: 76).

El mayor nodo que se observa en esta red de colaboración es el de Ecopetrol, que ocupa una posición estratégica manifiesta en su alto grado de desarrollo e integración. Los agregados de esta red probablemente están bajo la tutela de un grupo de investigadores bien estructurado; se detecta entre los agregados de este nodo una fuerte conexión. Los principales innovadores de esta red son Jorge Enrique Forero Sanabria, Gonzalo Leal Díaz, Guillermo Latorre Cortés y Olga Patricia Ortiz Cansino, quienes constituyen el centro de la misma. El tamaño de los enlaces indica una mayor cosolicitud 
de patentes. El nodo de la Pontificia Universidad Javeriana muestra un interesante nivel de patentamiento a través de la colaboración entre y con los innovadores. Se resalta el subnodo a partir de uno de sus agregados y la patentación con la Fundación Universidad del Norte, principalmente. El patentamiento a través de la colaboración de la Universidad Nacional de Colombia se caracteriza por la debilidad de las relaciones con sus agregados.

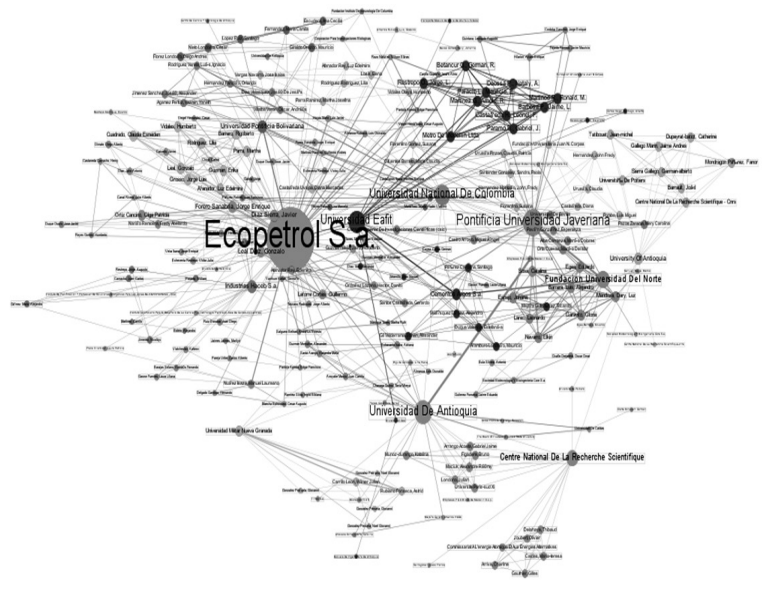

Figura 8. Redes de colaboración

En la red general se detectan agregados que se podrían denominar periféricos o podría tratarse de agregados que aún siguen siendo objeto de inversiones importantes en materia de innovación, y que se han visto marginados progresivamente llamando cada vez menos interés a su alrededor. Aparecen como redes que actúan débilmente con respecto a otras subredes y representan los márgenes de la red. Es el caso de la Universidad Militar Nueva Granada, cuya mayor densidad es con Wilmer Carrillo León y Astrid Rubiano Fonseca, y de Industrias HACEB S.A. y la subred compuesta por Luis Echeverría Afanador, Jorge Luis Granados, Humberto Videla, Claudia E. Cuadrado, Lilia Rodríguez, Martha Parra y Erika Guzmán, cuya densidad de las relaciones se muestra muy débil con respecto a otras subredes.

Se identifica, en el mismo sentido, el nodo de la Universidad de Antioquia entre cuyos agregados se encuentra el Centre National de la Recherche Scientifique; esta es una muestra del patentamiento colombiano en el exterior y de colaboración. 


\section{Discusión}

Se detecta una tendencia creciente de la actividad de patentación principalmente al final del periodo estudiado; no obstante, a pesar de que cada vez se incrementa el número de patentes en las bases de datos de patentes, los resultados generales del país no son los más convenientes frente a otros países, confirmándose lo anotado por Morales y Sifontes (2013) y Sánchez, Medina y León (2007).

Los resultados de la investigación son concordantes con los estudios de la OCDE (2016) según los cuales las empresas colombianas están siendo cada vez menos innovadoras y el gasto en actividades de $\mathrm{I}+\mathrm{D}$ es demasiado bajo (70 \% del PIB).

Se pone de manifiesto la teoría de la productividad patentométrica sesgada (Sánchez Guzmán, 1999: 42), según la cual la productividad de los inventores, bien sea personas naturales o instituciones, no es la misma. Parece que un innovador o empresa con mayor número de patentes puede tener mayor facilidad para producir otras y seguramente se le asignará mayor cantidad de recursos para desarrollar nuevas patentes; por consiguiente, siempre va a existir un pequeño grupo de innovadores y empresas muy productivas y otras que no lo serán de la misma manera.

Se evidencia el intento de los inventores por ser más competitivos y la cooperación universidad-empresa se presenta con muchas perspectivas; sin embargo, no se puede desconocer que el patentamiento y posicionamiento de los productos de patentes en el mercado tienen un costo relacionado con la adquisición de licencias o tecnologías, entre otros aspectos, que es posible que no estén al alcance de los inventores, por lo cual la alternativa es la realización de alianzas estratégicas con otras empresas para efectuar desarrollos conjuntos, como parte de la estrategia empresarial. Igualmente, no se puede desconocer que en Colombia no se pueden patentar los seres vivos ni los segundos usos de los medicamentos ya patentados, lo cual podría influir en la cantidad de patentes registradas. Tampoco se puede patentar el software, aunque sí puede ser registrado según la legislación de derechos de autor. La base de esta reglamentación está contemplada en la Decisión 486 de la Comunidad Andina de Naciones (CAN) y en el Decreto 2591 de 2000.

\section{CONCLUSIONES Y RECOMENDACIONES}

Se advierte la importancia del salto que Colombia ha dado en la inversión en actividades de ciencia y tecnología y su incidencia en el desarrollo y la competitividad del país y, por tanto, en la disminución de la brecha tecnológica 
con relación a otros países de la región, aun cuando no en la medida que se esperaría. La innovación tecnológica se constituye en la base de la competitividad del país y en una mayor integración de los productos, procesos y mercado, lo que determina la dinámica de patentación de los inventos de los colombianos en el periodo de estudio, principalmente a mediados cuando la dinámica cambia.

Se encontró que un porcentaje importante de patentes pertenecen a universidades como la Javeriana (8\%), la Universidad de Antioquia (6.5 \%), la Universidad Nacional de Colombia (7.4 \%), la Universidad Eafit (2.5\%) y la Universidad Militar (7.0 \%). Esta realidad pone de manifiesto el avance en la relación empresa-universidad para la generación de alianzas estratégicas que benefician el progreso tecnológico del país.

El progreso tecnológico a través del patentamiento muestra una apuesta explícita hacia el sector de las tecnologías para el manejo de residuos (141 patentes), preparaciones medicinales que contienen ingredientes orgánicos activos (145 patentes) y las caracterizadas por la forma física especial y generación de energía a través de fuentes de energía renovables en sus dos vertientes: la generación de energía y las fuentes de generación de la misma (87 patentes). Sin embargo, las patentes orientadas hacia el campo de preparaciones medicinales que contienen activos orgánicos, biocidas, repelentes de plagas y atrayentes o reguladores del crecimiento de las plantas que contienen compuestos heterocíclicos tienen gran importancia en el país (214 patentes).

Se detectaron áreas emergentes de patentación. A partir de 2009 las organizaciones o inventores se interesaron por inventos relacionados con preparaciones para tocador y patentamiento aislado en ingeniería genética, potenciando sus propias capacidades en $\mathrm{I}+\mathrm{D}$ y las de otras instituciones como las universidades.

La red de colaboración muestra que son numerosos los esfuerzos de patentamiento individual, más que de colaboración, que se realizan en el periodo analizado. Es importante resaltar que las invenciones de los colombianos no son necesariamente patrocinadas por empresas o instituciones de origen colombiano. En un buen número de los casos se trata de instituciones de carácter internacional como el Centre National de la Recherche Scientifique, perteneciente al nodo de la Universidad de Antioquia. 
La autora quiere agradecer a Sergio Cuellar Parrado y Matt Troyer por su colaboración en la investigación.

Artículo basado en los resultados del proyecto "Tendencias de innovación tecnológica que rigen los procesos y productos en Colombia durante el periodo 1991 - 2013 mediante el análisis de las patentes solicitadas y concedidas a colombianos" INP-2016-008218.

\section{REFERENCIAS}

Barceló, Miquel, Francesc Solé Parellada y Jaume Valls. 1992. “Tecnología y PME en España”. Economía Industrial 284: 127-136.

BID (Banco Interamericano de Desarrollo). 2014. “¿Cómo Repensar El Desarrollo Productivo?”, en Políticas e Instituciones Sólidas Para La Transformación Económica, Gustavo Crespi, Eduardo Fernández-Arias y Ernesto Stein (ed.), Washington: BID. https://publications.iadb.org/handle/11319/6634

Callon, M., J. P. Courtial y H. Penan. 1995. Cienciometría. El estudio cuantitativo de la actividad cientifica: de la bibliometría a la vigilancia tecnológica. Gijón, España: Ediciones Trea.

Camps, Diego. 2008. "Limitaciones de los indicadores bibliométricos en la evaluación de la actividad científica biomédica”. Colombia Médica 39 (1): 74-79.

Castellanos, Oscar Fernando, Andrés Mauricio León, Víctor Mauricio Montañez, Claudia Nelcy Jiménez y Freddy Abel Vargas. 2005. "El análisis de patentes como instrumento para la generación de conocimiento y de estrategia tecnológica”. Revista GTI 4 (9): 71-82.

CONPES (Consejo Nacional de Política Económica y Social). 2008. Política Nacional De Competitividad y Productividad.

Escorsa, Pere, Ramón Maspons y Joan Llibre. 2001. De la vigilancia tecnológica a la inteligencia competitiva. Madrid: Prentice Hall.

Goodman, Richard Arthur y Michael W. Lawless. 1994. Technology and Strategy: Conceptual Models and Diagnostics. Oxford University Press on Demand.

Huang, Cui, Jun Su, Xiang Xie, Xuanting Ye, Zhang Li, Alan Porter y Jiang Li. 2015. "A Bibliometric Study of China’s Science and Technology Policies: 1949-2010". Scientometrics 102 (2): 1521-1539.

Huang, Mu-Hsuan, Han-Wen Chang y Dar-Zen Chen. 2012. "The Trend of Concentration in Scientific Research and Technological Innovation: A Reduction of the Predominant Role of the US in World Research \& Technology”. Journal of Informetrics 6 (4): 457-468.

Lucio, Jorge, Diana Lucio-Arias, Mónica Salazar, Cristhian Ruiz, Edgar Bueno, Jose Montes, Juliana Velandia et al. 2013. Observando el sistema colombiano de ciencia, tecnología e innovación: sus actores y sus productos. Colombia: Observatorio Colombiano de Ciencia y Tecnología. 
Montes Hincapié, Juan Manuel, Joaquín Lloveras Macià, Pere Escorsa i Castells y Elicet Cruz Jiménez. 2009. "Análisis de patentes en el sector de los aceites esenciales como estrategia para impulsar el desarrollo regional en Antioquia”. XIII Seminario Latino-iberoamericano de Gestión Tecnológica ALTEC, Cartagena de Indias

Morales, R., y D. Sifontes. 2013. "Reporte de la Actividad Innovadora de América Latina: Un Estudio de Patentes”. VIII Congreso de Indicadores de Ciencia y Tecnología.

OCDE (Organización para la Cooperación y el Desarrollo Económicos). 2016. Estudios de la OCDE de las Políticas de Innovación: Colombia. OECD Publishing. https://www.oecd.org/sti/inno/colombia-innovation-review-assessment-and-recommendations-spanish.pdf.

OCDE. 2003. Manual de Frascati. Propuesta de norma práctica para encuestas de investigación y desarrollo experimental. París: Fundación Española para la Ciencia y la Tecnología (FECYT).

OMPI (Organización Mundial de la Propiedad Intelectual). 2017. Manual de la OMPI de redacción de solicitudes de patente. Ginebra: OMPI.

Orozco, Luis Antonio y Diego Andrés Chavarro. 2010. "Robert K. Merton (19102003). La ciencia como institución”. Revista de Estudios Sociales (37): 143-162.

Ortiz, Sara y Álvaro R. Pedroza Zapata. 2006. “¿Qué es la gestión de la innovación y la tecnología (GInnT)?” Journal of Technology Management EInnovation 1 (2): 64-82.

Porter, Alan L. y Scott W. Cunningham. 2004. Tech Mining: Exploiting New Technologies for Competitive Advantage. Hoboken, New Jersey: John Wiley \& Sons.

Sánchez Guzmán, M. V. 1999. "Patentometría. Herramienta para el análisis de oportunidades tecnológicas”. Tesis, Doutorado em Gerência de Información Tecnológica, Facultad de Economía, Universidad de La Habana, Cuba.

Sánchez, Jenny Marcela, Javier E. Medina y Andrés Mauricio León. 2007. "Publicación internacional de patentes por organizaciones inventores de origen colombiano". Cuadernos de Economía 26 (47): 247-270.

Spinak, Ernesto. 1998. "Indicadores cienciométricos”. Ciência da Informação 27 (2).

Trejos, Alberto, Graciana Rucci, Marina Bassi, Alessandro Maffioli, Jorge Cornick, Alejandro Rasteletti, Carlo Pietrobelli, Fernando de Olloqui, Gabriel Casaburi y Manuel R. Agosin. 2014. ¿Cómo repensar el desarrollo productivo? Políticas e instituciones sólidas para la transformación económica. Washington: Inter-American Development Bank. https://publications.iadb.org/handle/11319/6634

Zhou, Xiao, Yi Zhang, Alan L. Porter, Ying Guo y Donghua Zhu. 2014. "A Patent Analysis Method to Trace Technology Evolutionary Pathways". Scientometrics 100 (3): 705-721.

Para citar este texto:

Gómez Hurtado, Rosa Elena. 2018. “Tendencias de la innovación tecnológica en Colombia 1991-2013 a partir del análisis de patentes”. Investigación Bibliotecológica: archivonomía, bibliotecología e información 32 (77): 133-150. http://dx.doi.org/10.22201/iibi.24488321xe.2018.77.57859 